\title{
NORMS OF HANKEL OPERATORS ON A BIDISC
}

\author{
TAKAHIKO NAKAZI
}

(Communicated by Paul S. Muhly)

\begin{abstract}
In the Hardy space on the bidisc $T^{2}$, if $\phi$ is a bounded function in the Lebesgue space and if its Fourier series vanishes on half of $\mathbf{Z}^{2}$, then the norm of the Hankel operator $H_{\phi}$ is equal to the quotient norm of $\phi$ by the Hardy space $H^{\infty}\left(T^{2}\right)$.
\end{abstract}

Let $\sigma$ denote the Haar measure of the torus $T^{2}$, the distinguished boundary of the unit bidisc $U^{2}$ in the space of 2 complex variables $(z, w)$. Put $\mathbf{Z}_{+}^{2}=$ $\left\{(m, n) \in \mathbf{Z}^{2} ; m \geq 0\right.$ and $\left.n \geq 0\right\}$. For $1 \leq p \leq \infty, L^{p}=L^{p}\left(T^{2}, \sigma\right)$ denotes the Lebesgue space and $H^{p}=H^{p}\left(T^{2}, \sigma\right)=\left\{f \in L^{p} ; \hat{f}(m, n)=0\right.$ if $(m, n) \notin$ $\left.\mathbf{Z}_{+}^{2}\right\}$. That is, $H^{p}$ denotes the usual Hardy spaces on the bidisc. Let $K_{0}^{p}=$ $\left\{f \in L^{p} ; \hat{f}(m, n)=0\right.$ if $\left.(-n,-m) \in \mathbf{Z}_{+}^{2}\right\}$.

For $\phi \in L^{\infty}$, the Hankel operator determined by $\phi$ is

$$
H_{\phi}=(I-P) M_{\phi} \mid H^{2}
$$

and the Toeplitz operator determined by $\phi$ is

$$
T_{\phi}=P M_{\phi} \mid H^{2},
$$

where $P: L^{2} \rightarrow H^{2}$ is the orthogonal projection and $M_{\phi}$ is the multiplication operator on $L^{2}$ associated with $\phi$.

In the classical Hardy space $H^{2}(T)$ on the unit disc, the norms of Hankel operators $H_{\phi}$ are equal to the quotient norms of the symbols $\phi$ by $H^{\infty}(T)$ ([7], [8, pp. 4-6]). This is called Nehari's theorem. The author [6] gave a generalization of Nehari's theorem to the Hardy space $H^{2}\left(T^{2}\right)$ on the bidisc. The theorem is analogous to Nehari's theorem but does not give the norm of $H_{\phi}$ using the symbol $\phi$. For the Hardy space on the unit ball of $\mathbf{C}^{n}$, it is known that the norm of a Hankel operator $H_{\phi}$ is equivalent to the BMO norm

Received by the editors March 1, 1989.

1980 Mathematics Subject Classification (1985 Revision). Primary 47A30, 47B35; Secondary $46 \mathrm{~J} 15,42 \mathrm{~B} 30$.

Key words and phrases. bidisc, Hardy space, Hankel operator, norm.

This research was partially supported by Grant-in-Aid for Scientific Research, Ministry of Education. 
of $\phi$ (cf. [2], [9]). However we do not know whether the same theorem is valid or not for that on the polydisc. In this paper, for the bidisc we show that if $\phi \in L^{\infty}$ is a function whose Fourier series vanish on the half of $\mathbf{Z}^{2}$, then $\left\|H_{\phi}\right\|=\left\|\phi+H^{\infty}\right\|$.

In the classical Hardy space, the essential norms of Hankel operators are the quotient norms of the symbols by $H^{\infty}+C(T)$, where $C(T)$ denotes the set of all continuous functions on the unit circle (cf. [8, p. 6]). Hence there exist many nonzero compact Hankel operators. However Curto, Muhly and Nakazi [3] showed that there does not exist any nonzero compact Hankel operator in the bidisc Hardy space. In this paper, roughly speaking, we show that if $\phi \in L^{\infty}$ is a function whose Fourier series vanish on the half of $\mathbf{Z}^{2}$, then $\left\|H_{\phi}\right\|_{e}=\left\|H_{\phi}\right\|$ where $\left\|H_{\phi}\right\|_{e}$ denotes the essential norm. Moreover we use results about Hankel operators to study the invertibility of Toeplitz operators.

An order relation can be introduced in $\mathrm{Z}^{2}$. Let $L_{r}$ be a line with rational slope $r$ in the plane. $S_{r}$ denotes all lattice points on one side of $L_{r}$, together with those on the right side ray of $L_{r}$ from the origin. When $L$ is a real axis, that is, $L=L_{0}$, then $S_{0}=\{(m, 0): m>0\} \cup\{(m, n): m>0\}$. When $L$ is an imaginary axis, that is, $L=L_{-\infty}$, then $S_{-\infty}=\{(m, n): m>0\} \cup\{(0, n): n>$ $0\}$. This order is non-archimedean, and $\mathbf{Z}^{2}$ has a smallest positive element $\left(m_{0}, n_{0}\right)$ in $S_{r}$. We assume that $S_{r}$ contains $\mathbf{Z}_{+}^{2}$, that is, $-\infty \leq r \leq 0$. When $-\infty<r<0,\left|m_{0}\right|$ and $\left|n_{0}\right|$ have no common factor except 1 and $r=n_{0} / m_{0}$, and $\left(m_{1}, n_{1}\right)=(0,1)$. When $r=0,\left(m_{0}, n_{0}\right)=(1,0)$ and let $\left(m_{1}, n_{1}\right)=(0,1)$. When $r=-\infty,\left(m_{0}, n_{0}\right)=(0,1)$ and let $\left(m_{1}, n_{1}\right)=(1,0)$. For each half plane $S_{r}$, put

$$
Z=Z_{r}=z^{m_{0}} w^{n_{0}}
$$

and

$$
W=W_{r}=z^{m_{1}} w^{n_{1}}
$$

Hence $Z_{0}=W_{-\infty}=z$ and $W_{0}=Z_{-\infty}=w$, and if $-\infty<r<0$ then $W_{r}=w$.

For each $r$ with $-\infty \leq r \leq 0$, put $\mathbf{H}_{r}^{p}=$ the norm closure of $\cup_{j=-\infty}^{\infty} Z_{r}^{j} H^{p}$ in $L^{p}$ if $1 \leq p<\infty$ and $\mathbf{H}_{r}^{\infty}=$ the weak ${ }^{-*}$ closure of $\cup_{j=-\infty}^{\infty} Z_{r}^{j} H^{\infty}$ in $L^{\infty}$. $\mathscr{L}_{r}^{p}$ and $\mathscr{H}_{r}^{p}$ denote the norm closure of the set of trigonometric polynomials and analytic polynomials, respectively, of $Z_{r}$ in $L^{p}$ if $1 \leq p<\infty . \mathscr{L}_{r}^{\infty}$ and $\mathscr{H}_{r}^{\infty}$ denote the weak ${ }^{-*}$ closure. Then

$$
\mathbf{H}_{r}^{p}=\mathscr{L}_{r}^{p}+\mathscr{L}_{r}^{p} W+\cdots+\mathscr{L}_{r}^{p} W^{n-1}+W^{n} \mathbf{H}_{r}^{p} .
$$

Let $E$ be a conditional expectation from $\mathbf{H}_{r}^{\infty}$ onto $\mathscr{L}_{r}^{\infty}$. Then $E$ is multiplicative on $\mathbf{H}_{r}^{\infty}$ and $\mathbf{H}_{r}^{\infty}+{\overline{W \mathbf{H}_{r}}}_{r}^{\infty}$ is weak ${ }^{-*}$ dense in $L^{\infty}$. Hence $\mathbf{H}_{r}^{\infty}$ is an 
extended weak ${ }^{-*}$ Dirichlet algebra with respect to $E$ (see [5]). Thus Theorems 4 and $4^{\prime}$ in [5] imply

Lemma 1. Let $h \in \mathbf{H}_{r}^{1}$ and let $\varepsilon>0$ be given. Then there is an $f \in \mathbf{H}_{r}^{2}$, $\|f\|_{2} \leq\|h\|_{1}$, and a $g \in \mathbf{H}_{r}^{2},\|g\|_{2} \leq\|h\|_{1}+\varepsilon$, such that $h=f g$.

The proof is almost the same as the proof of Lemma 2.1 in [4].

Theorem 1. If $\phi$ is a function in $L^{\infty}$, then

$$
\left\|\phi+H^{\infty}\right\| \geq\left\|H_{\phi}\right\| \geq \sup \left\{\left\|\phi+\mathbf{H}_{r}^{\infty}\right\| ;-\infty \leq r \leq 0\right\} .
$$

Proof. It is sufficient to show that $\left\|H_{\phi}\right\| \geq\left\|\phi+\mathbf{H}_{r}^{\infty}\right\|$ for any $r$ with $-\infty \leq$ $r \leq 0$. Fix $r$ with $-\infty \leq r \leq 0$. Since $Z^{j} \mathbf{H}_{r}^{2}=\mathbf{H}_{r}^{2}$ for any integer $j$, $H^{2} \times W \mathbf{H}_{r}^{2}=Z^{j} H^{2} \times W \mathbf{H}_{r}^{2}$ and hence

Hence,

$$
H^{2} \times K_{0}^{2} \supset\left\{\cup_{j=-\infty}^{\infty} Z^{j} H^{2}\right\} \times W \mathbf{H}_{r}^{2} .
$$

$$
\begin{aligned}
&\left\|H_{\phi}\right\|=\sup \left\{\left|\int \phi f g d \sigma\right|: f \in H^{2}, g \in K_{0}^{2},\right. \\
&\left.\|f\|_{2} \leq 1 \text { and }\|g\|_{2} \leq 1\right\} \\
& \geq \sup \left\{\left|\int \phi f g d \sigma\right|: f \in \cup_{j=-\infty}^{\infty} Z^{j} H^{2}, g \in W \mathbf{H}_{r}^{2},\right. \\
&\left.\quad\|f\|_{2} \leq 1 \text { and }\|g\|_{2} \leq 1\right\} \\
&=\sup \left\{\left|\int \phi f g d \sigma\right|: f \in \mathbf{H}_{r}^{2}, g \in W \mathbf{H}_{r}^{2},\right. \\
&\left.=\left\|\phi+\mathbf{H}_{r}^{\infty}\right\| . \quad\|f\|_{2} \leq 1 \text { and }\|g\|_{2} \leq 1\right\}
\end{aligned}
$$

We used Lemma 1 in the last equality (cf. [4, Corollary 2.1.1.]).

Lemma 2. If $\phi \in \bar{W}_{r}^{\infty}$ then $\left\|\phi+\mathbf{H}_{r}^{\infty}\right\|=\left\|\phi+H^{\infty}\right\|$.

Proof. By duality, it is sufficient to show that

$$
\begin{aligned}
\sup \left\{\left|\int \phi f d \sigma\right|: f \in K_{0}^{1},\|f\|_{1}\right. & \leq 1\} \\
& =\sup \left\{\left|\int \phi F d \sigma\right|: F \in W \mathbf{H}_{r}^{1},\|F\|_{1} \leq 1\right\} .
\end{aligned}
$$

Let $Q$ be an orthogonal projection from $L^{2}$ onto $W \mathbf{H}_{r}^{2}$. If $\phi \in{\overline{W \mathbf{H}_{r}}}_{r}^{\infty}$, then for any $f \in K_{0}^{2}$,

$$
\int \phi f d \sigma=\int \phi Q(f) d \sigma .
$$

Since $Q K_{0}^{2}=W \mathbf{H}_{r}^{2}$, the two supremums above are equal because $K_{0}^{2}$ is dense in $K_{0}^{1}$ and $\mathbf{H}_{r}^{2}$ is dense in $\mathbf{H}_{r}^{1}$. 
Theorem 2. For any $r$ with $-\infty \leq r \leq 0$, if $\phi$ is in $\overline{W \mathbf{H}}_{r}^{\infty}+H^{\infty}$, then

$$
\left\|H_{\phi}\right\|=\left\|\phi+H^{\infty}\right\| .
$$

The proof is clear by Theorem 1 and Lemma 2 .

For any $r$ with $-\infty \leq r \leq 0$, we want to know about $\left\|H_{\phi}\right\|$ for $\phi$ in $\mathscr{L}_{r}^{\infty}$. The following proposition answers the request only when $r=0$ or $-\infty$.

Proposition 3. Put $r=0$ or $-\infty$. If $\phi$ is in $\mathscr{L}_{r}^{\infty}+H^{\infty}$ then

$$
\left\|H_{\phi}\right\|=\left\|\phi+H^{\infty}\right\| .
$$

Proof. For any $r, \mathscr{H}_{r}^{p}$ is isometrically isomorphic to the classical Hardy space $H^{p}(T)$. Hence by Nehari's theorem (cf. [8, p. 11]),

$$
\begin{aligned}
\left\|H_{\phi}\right\| & \geq \sup \left\{\left|\int \phi f g d \sigma\right| ; f \in \mathscr{H}_{r}^{2}, g \in \mathscr{H}_{r}^{2},\right. \\
& \left.\|f\|_{2} \leq 1 \text { and }\|g\|_{2} \leq 1\right\} \\
& =\left\|\phi+\mathscr{H}_{r}^{\infty}\right\| .
\end{aligned}
$$

If $r=0$ or $-\infty, H^{\infty} \supset \mathscr{H}_{r}^{\infty}$ and hence the proposition follows.

By Theorem 1, we can ask whether $\left\|H_{\phi}\right\|=\sup _{r}\left\|\phi+\mathbf{H}_{r}^{\infty}\right\|$ is true or not. By Theorem 2, if $\phi \in{\overline{W \mathbf{H}_{r}}}_{r}^{\infty}+H^{\infty}$ then $\left\|H_{\phi}\right\|=\left\|\phi+\mathbf{H}_{r}^{\infty}\right\|$. However even if $\phi \in \mathscr{L}_{r}^{\infty}$ for $r=0$ or $-\infty$, we do not know whether this equality holds. If $\phi \in \overline{\mathscr{H}}_{r}^{\infty}+\mathscr{H}_{r}^{\infty}$ then $\phi-\hat{\phi}(0,0) \in \overline{W H}_{s}^{\infty}$ for some $s$ with $-\infty \leq s \leq 0$. Hence $\left\|H_{\phi}\right\|=\left\|\phi+\mathbf{H}_{s}^{\infty}\right\|$.

Now we will give two corollaries about essential norms of Hankel operators and Toeplitz operators.

Corollary 1. Put $r=0$ or $-\infty$. If $\phi$ is in $\overline{W \mathbf{H}}_{r}^{\infty}+H^{\infty}$ then

$$
\left\|H_{\phi}\right\|_{e}=\left\|H_{\phi}\right\|=\left\|\phi+H^{\infty}\right\| .
$$

Proof. By Theorem 2 and Lemma 2,

$$
\left\|H_{Z^{n} \phi}\right\|=\left\|Z^{n} \phi+H^{\infty}\right\|=\left\|\phi+H^{\infty}\right\|
$$

because $Z^{n} \phi \in{\overline{W \mathbf{H}_{r}}}_{r}+H^{\infty}$. Since $Z=z$ and $W=w$ for $r=0$, and $Z=w$ and $W=z$ for $r=-\infty$, both $T_{Z}$ and $T_{W}$ are unilateral shift operators on $H^{2}$ with infinite multiplicities. By the proof for classical Hardy space (cf. [1]), the theorem follows.

Corollary 2. Let $\phi$ be a unimodular function in $L^{\infty}$.

(1) For any fixed $r$ with $-\infty \leq r \leq 0$, suppose $\phi$ is in $\overline{W \mathbf{H}}_{r}^{\infty}+H^{\infty}$. Then $T_{\phi}$ is left invertible if and only if $\left\|\phi+H^{\infty}\right\|<1$.

(2) For $r=0$ or $-\infty$, suppose $\phi$ is in $\mathscr{L}_{r}^{\infty}$. Then $T_{\phi}$ is left invertible if and only if $\left\|\phi+H^{\infty}\right\|<1$.

(3) For $r=0$ or $-\infty$, suppose $\phi$ is in $\overline{W \mathbf{H}}_{r}^{\infty}$. Then $T_{\phi}$ is left Fredholm if and only if $\left\|\phi+H^{\infty}\right\|<1$. 
Proof. Using the well known equality

$$
T_{\phi}^{*} T_{\phi}+H_{\phi}^{*} H_{\phi}=I
$$

we can show (1) and (2) by Theorem 2 and Theorem 3, respectively, and (3) by Theorem 4.

\section{REFERENCES}

1. S. Axler, I. D. Berg, N. Jewell and A. Shields, Approximation by compacteperaters and the space $H^{\infty}+C$, Ann. of Math. 109 (1979), 601-612.

2. R. R. Coifman, R. Rochberg and G. Weiss, Fätorizätion theorens for Hardy spices inseveral variables, Ann. of Math. 103 (1976), 611-635."

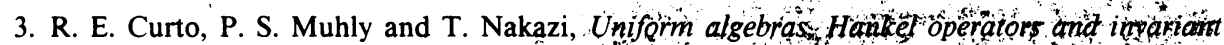
subspaces, Oper. Theory: Adv. Appl. 17 (1986), $1092119, \cdots$

4. R. E. Curto, P. S. Muhly, T. Nakazi and J. Xia, Hankel operators and uniform algebros Archiv der Math. 43 (1984), 440-447.

5. T. Nakazi, Extended weak ${ }^{-*}$ Dirichlet algebras, Pacific J. Math. 81 (1979), 493-513.

6. __ Norms of Hankel operators and uniform algebras, Trans. Amer. Math. Soc. 299 (1987), 573-580.

7. Z. Nehari, On bounded bilinear forms, Ann. of Math. 65 (1957), 153-162.

8. S. C. Power, Hankel operators on Hilbert space, Research Notes in Math. 64, Pitman, Boston, 1982.

9. A. Uchiyama, On the compactness of operators of Hankel type, Tohoku Math. J. 30 (1978), 163-171.

Department of Mathematics, Faculty of Science, Hokkaido University, Sapporo 060, JAPAN 